\title{
High precision neutron inelastic cross sections on ${ }^{16} \mathrm{O}$
}

\author{
Boromiza Marian ${ }^{1, *}$, Borcea Catalin ${ }^{1}$, Dessagne Philippe ${ }^{2}$, Henning Gregoire $^{2}$, Kerveno Mäelle ${ }^{2}$, Negret Alexandru ${ }^{1}$, \\ Nyman Markus ${ }^{3}$, Olacel Adina ${ }^{1}$, and Plompen Arjan ${ }^{3}$ \\ ${ }^{1}$ Horia Hulubei National Institute for Physics and Nuclear Engineering, Reactorului 30, 077125 Bucharest-Măgurele, Romania \\ ${ }^{2}$ Université de Strasbourg, CNRS, IPHC UMR 7178, F- 67000 Strasbourg, France \\ ${ }^{3}$ European Commission, Joint Research Centre, Retieseweg 111, B- 2440 Geel, Belgium
}

\begin{abstract}
This work reports partial results of a $(\mathrm{n}, \mathrm{n} \gamma)$ measurement on ${ }^{16} \mathrm{O}$. The $\gamma$ rays of interest from the inelastic channel were detected using the Gamma Array for Inelastic Neutron Scattering (GAINS) spectrometer at the Geel Electron Linear Accelerator (GELINA) neutron source. A very thick (32.30(4) $\mathrm{mm}$ ) $\mathrm{SiO}_{2}$ target was used. The main goal was to determine the angle-integrated $\gamma$-production cross section for the most important transitions. In this work we report the results for the main ${ }^{16} \mathrm{O}$ transition and we emphasize a consistency check aiming to ensure data reliability. Our results are compared with theoretical calculations performed using the TALYS 1.8 code and with previously reported experimental data.
\end{abstract}

\section{Introduction}

In many countries nuclear reactors play an important role in energy production due to their evident advantages as compared with the standard options: limited pollution and in particular no carbon emissions, high energy density of the fuel and high reliability. Concerns related to nuclear energy include safety, nuclear waste, limited fuel, economic viability and danger of proliferation.

Presently, the scientific community aims at developing a new type of nuclear reactor - Generation IV fast and thermal reactors - that will address many of these issues ( [1] and the references therein). These reactors are able to transmute the minor actinides from the fuel, thus shortening the time nuclear waste remains highly radioactive. Also, they will be fuelled by more abundant isotopes $\left({ }^{238} \mathrm{U}\right.$ or ${ }^{232} \mathrm{Th}$ ), which will ensure sufficient fuel supply for a very long time. Contrary to ${ }^{235} \mathrm{U}$, the fission cross section on ${ }^{238} \mathrm{U}$ starts to become relevant only above $1 \mathrm{MeV}$ neutron energy [2]. Therefore, many Generation IV reactors will make use of fast-neutron induced fission to produce energy (i.e. no neutron moderation is needed).

From a technological point of view however, the design of Generation IV reactors requires very low uncertainty reaction data for an extended incident energy range (mainly above the thermal region), in particular for the inelastic channel.

The most abundant stable oxygen isotope is ${ }^{16} \mathrm{O}$ (99.75\% [3]) which is an important structural component due to presence of oxides in the reactor environment. Also, oxygen is present in some type of nuclear fuels like UOX or MOX. This is the motivation for including ${ }^{16} \mathrm{O}$ on the list of the six isotopes evaluated by the CIELO collaboration [4] and also on the High Priority Request List (HPRL)

*e-mail: marian.boromiza@nipne.ro of Nuclear Energy Agency (NEA) [5]. The HPRL solicits data with a 3-5\% uncertainty for the neutron-induced inelastic channel [5].

One of the heat-producing mechanisms in a nuclear reactor is given by the $\gamma$ rays following inelastic neutron scattering. In this context, an accurate knowledge of the neutron-induced inelastic $\gamma$-production cross sections on ${ }^{16} \mathrm{O}$ is important.

Here we will only present the results extracted for the first ${ }^{16} \mathrm{O} \gamma$ ray while the cross section for the other transitions and the total inelastic cross section will be reported elsewhere. We will also describe in detail a consistency check aiming to ensure the reliability of the data measured using the GELINA-GAINS setup.

\section{Experimental setup}

The present experiment was performed using the GELINA-GAINS facility of the European Commission Joint Research Center in Geel, Belgium (see Fig. 1) [69]. It consists of a (pulsed) white neutron source coupled with $\gamma$ spectroscopy based on HPGe detectors and time-offlight technique for determining the incident energy. The detector signals were digitized by ACQIRIs DC440 digitizers with a sampling frequency of $420 \mathrm{MHz}$ and an amplitude range of 12 bits. A fission chamber with ${ }^{235} \mathrm{U}$ deposits was used for incident flux monitoring and it was read-out by conventional electronics [10]. A very thick (32.30(4) mm) $\mathrm{SiO}_{2}$ sample with a diameter of 76.26(4) mm was used which was irradiated for a total of $472 \mathrm{~h}$.

\section{Data analysis procedure}

After the data analysis the primary-extracted quantity is the differential $\gamma$-production cross section at two angles: 


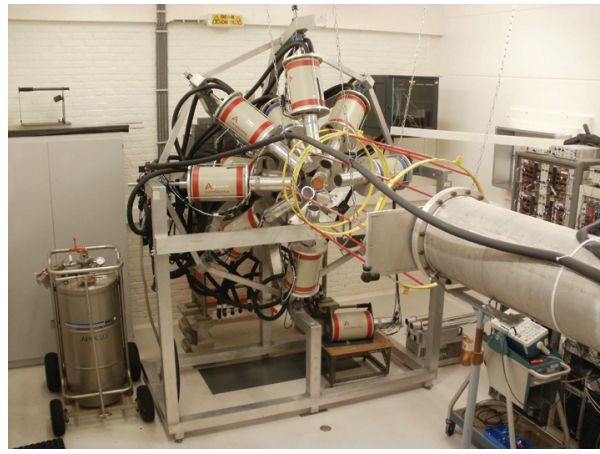

Figure 1. (Colour online) The GAINS spectrometer used during our experiment. It consisted of twelve HPGe detectors with $100 \%$ relative efficiency placed at $110^{\circ}, 125^{\circ}$ and $150^{\circ}$ relative to the incident neutron beam.

$110^{\circ}$ and $150^{\circ}$ while the one at $125^{\circ}$ is used only for cross checking our data. These detection angles allow for a very precise angular integration procedure using the Gaussian Quadrature Method and a Legendre polynomials series expansion of the differential cross sections. The procedure for determining the cross sections reported here (including the detailed formulae) is described extensively in Refs. [8, 11-14].

\section{Results and discussion}

Figure 2 displays the partial level scheme of ${ }^{16} \mathrm{O}$, adapted from Ref. [15]. The first excited level decays through a totally converted E0 transition. We employ $\gamma$ spectroscopy techniques, hence, we could not detect this transition. Given the very low abundance of the other stable oxygen isotopes [3], no $\gamma$ peaks corresponding to ${ }^{17} \mathrm{O}$ or ${ }^{18} \mathrm{O}$ were observed in our spectra. Also, the ${ }^{17} \mathrm{O}(\mathrm{n}, 2 \mathrm{n} \gamma)^{16} \mathrm{O}$ polluting contributions in the peaks of interest from ${ }^{16} \mathrm{O}$ were completely negligible $\left({ }^{17} \mathrm{O}\right.$ has an abundance of only $0.038 \%)$.

Figure 3 displays our results for the main ${ }^{16} \mathrm{O}$ transition. Other experimental values were measured by Nelson et al. [16], Dickens et al. [17], Orphan et al. [18] and Besotosnyj et al. [19]. When comparing our results with the ones reported by Nelson et al., the agreement between the two data sets is very good in the entire incident energy range. Both experiments report neutron-induced cross sections with good incident neutron energy resolution. The two data sets are complementary considering that the Nelson et al. data display a much better statistics at high neutron energies where the GELINA neutron flux is very small. However, the present data have a much better neutron energy resolution (around $35 \mathrm{keV}$ versus $110 \mathrm{keV}$ at $10 \mathrm{MeV}$ incident energy [16]) and report more cross section points below $10 \mathrm{MeV}$ incident energy.

The TALYs 1.8 reaction code, in the default settings, predicts well the shape of our experimental cross section while the absolute values are generally underestimated (see Fig. 3).

The neutron-induced inelastic $\gamma$-production cross section for the $6128.6-\mathrm{keV}$ transition is reported with a total

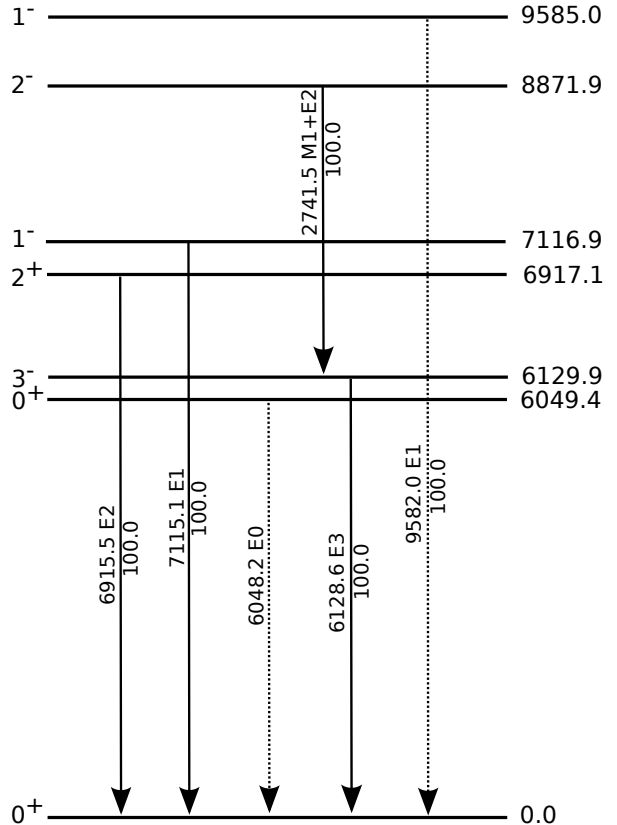

Figure 2. A partial level scheme of ${ }^{16} \mathrm{O}$, adapted from Ref. [15]. We extracted cross sections for all the transitions drawn with a continuous line. However, in the present work we report only the results for the $6128.6-\mathrm{keV}$ transition while the data for the 6915.5-, 7115.1- and 2741.5-keV $\gamma$ rays will be presented elsewhere. The level and $\gamma$ energies are given in $\mathrm{keV}$.

relative uncertainty under $6 \%$ for most of the incident energy range (see Fig. 3, panel a), which is close to the one solicited by HPRL of NEA [5] (see Section 1).

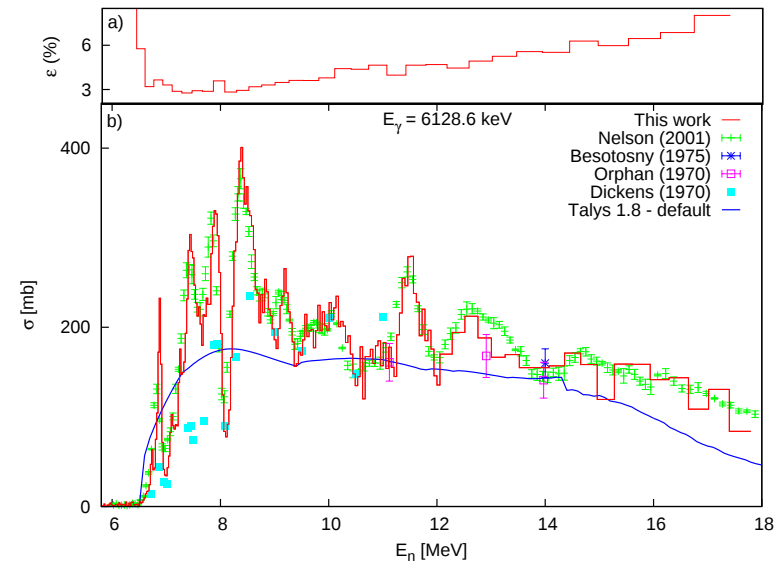

Figure 3. (Colour online) The inelastic angle-integrated $\gamma$ production cross section for the $6128.6-\mathrm{keV}$ transition in ${ }^{16} \mathrm{O}$ obtained in the present work (panel b) with its associated relative uncertainty (panel a). It is compared with other available experimental data and with TALYs 1.8 model calculations performed using the default settings.

\section{Data consistency checks of the GELINA-GAINS setup}

Section 2 mentioned that we used a compound $\left(\mathrm{SiO}_{2}\right)$ target. This had a two-fold purpose. The most important goal 
was to determine the neutron-induced inelastic cross sections on ${ }^{16} \mathrm{O}$. Then, to extract and cross check also the ${ }^{28} \mathrm{Si}$ data considering that the inelastic cross sections on this nucleus were previously measured by Negret et al. using the GAINS spectrometer [20]. The most relevant difference between the two measurements was the target: ${ }^{n a t} \mathrm{Si}(\mathrm{Ne}-$ gret et al.) versus $\mathrm{SiO}_{2}$ (present work).
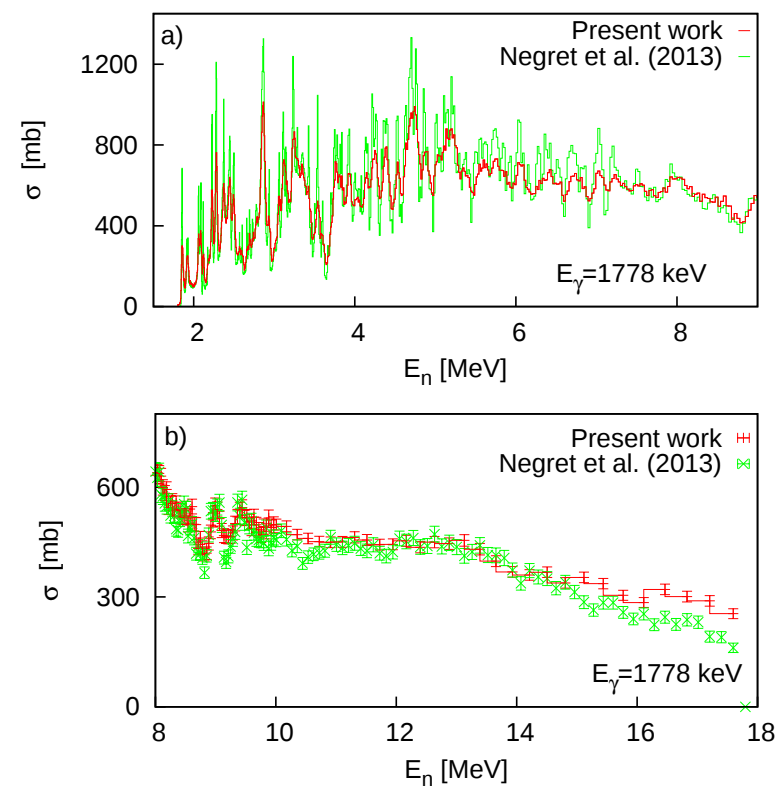

Figure 4. (Colour online) The $\gamma$-production cross section of the main transition in ${ }^{28} \mathrm{Si}$ measured in the two experiments mentioned in the main text (with quartz versus ${ }^{\text {nat }} \mathrm{Si}$ targets). Below $15 \mathrm{MeV}$ the agreement is very good. The results start to diverge above $15 \mathrm{MeV}$, with a maximum difference of $\approx 15-20 \%$ in the $15-18 \mathrm{MeV}$ range (see panel b). The two panels highlight the compound nucleus resonances and the high energy regions.

The comparison between the two experiments for the main transition in ${ }^{28} \mathrm{Si}(1778.9 \mathrm{keV})$ is displayed in Fig. 4. Notably, the present measurement displays a slightly worse neutron energy resolution. The agreement between the two cross sections is very good up to around $15 \mathrm{MeV}$ after which they start to diverge. The largest difference above $15 \mathrm{MeV}$ is around $15-20 \%$. The deviation looks to be energy dependent. The FC and $\gamma$ yields and the multiple scattering correction factor (MSC) depend on the incident energy [8, 14]. These three quantities and all the relevant reaction channels on ${ }^{16} \mathrm{O}$ and ${ }^{27} \mathrm{Al}$ were carefully checked for parasitic contributions. We note that the MSC had values ranging from 3-15\% depending on the transition and on the incident energy. Considering the much thicker target than the one of Ref. [20], the present experiment could have a higher aluminium-induced background component coming from the neutrons scattered by the sample and ending up in the GAINS aluminium frame or detector end caps. No contribution from either ${ }^{16} \mathrm{O}$ and ${ }^{27} \mathrm{Al}$ was found that would explain the difference observed in Fig. 4.

We mention that in the present experiment the detector preamplifier gain was changed from $500 \mathrm{mV} / \mathrm{MeV}$ to $100 \mathrm{mV} / \mathrm{MeV}$ to access the high $\gamma$ energy range neces- sary for detecting the ${ }^{16} \mathrm{O}$ transitions. Unfortunately, this change drastically affected the $\gamma$ energy resolution: a typical value around $6-8 \mathrm{keV}$ (at $1.3 \mathrm{MeV}$ ) was observed. This value is to be compared with the one of Ref. [20], which was around $2-3 \mathrm{keV}$.

Figure 5 displays a region in the $\gamma$-ray spectrum around the main ${ }^{28} \mathrm{Si}$ transition in the two experiments for two detectors placed at the same angle. The corresponding $\gamma$ energy resolutions and the integration limits of the 1778.9$\mathrm{keV}$ peak employed during the data analysis can be seen in the two cases. On the left side of the $1778.9-\mathrm{keV}$ transition there are two peaks: at $\approx 1720 \mathrm{keV}$ (from ${ }^{27} \mathrm{Al}$ and ${ }^{30} \mathrm{Si}$ ) and at $1764.4 \mathrm{keV}$ (from ${ }^{214} \mathrm{Bi}$ - natural background). On the right side there is a $1793.8-\mathrm{keV}$ peak corresponding to a transition originating from ${ }^{29} \mathrm{Si}$. The poor energy resolution of the present experiment combined with the integration limits shown in Fig. 5 resulted in the inclusion of the 1764.4- and the 1793.8-keV peaks in the integrated area of the $1778.9-\mathrm{keV}$ transition. On the other hand, the much better energy resolution of the Ref. [20] experiment allowed the discrimination between these three $\gamma$ peaks when the same integration was performed.

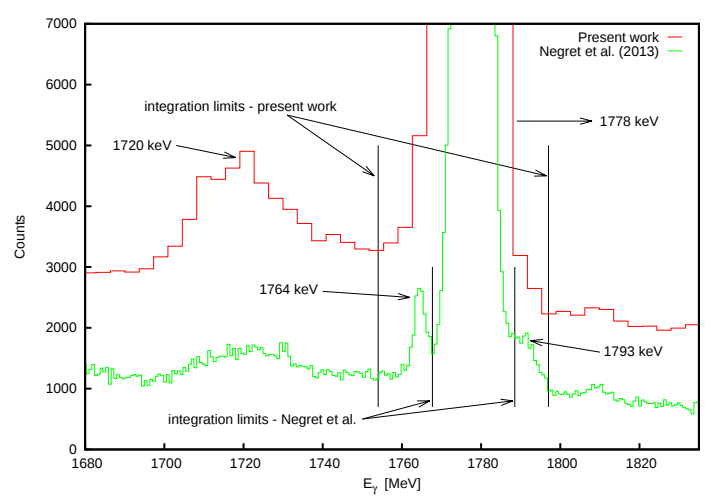

Figure 5. (Colour online) The $\gamma$ ray spectra, highlighting the main transition (1778.9 keV) and its neighbouring peaks, corresponding to the two compared experiments discussed in the text. The integration limits of the 1778.9-keV transition used during data analysis are indicated in both cases. They show that in the present work, due to the energy resolution deterioration and unlike the case of Ref. [20], the areas of the 1764.4- and the 1793.8$\mathrm{keV}$ neighbouring peaks were included in the one corresponding to the transition of interest from ${ }^{28} \mathrm{Si}$.

Figure 6 plots the amplitude spectrum of one HPGe detector used in the experiment of Ref. [20] but corresponding to neutrons with energies only in the $E_{n}=14-18 \mathrm{MeV}$ range (i.e. the energy region where the two compared cross sections differ by $\approx 15-20 \%$ - see Fig. 4 ).

The integration of the three $\gamma$ peaks from Fig. 6 gives the ratio:

$$
\left(A_{1793}+A_{1764}\right) /\left(A_{1778}\right) \simeq 14-18 \%
$$

where $A$ is the peak area. This value is consistent with the difference seen in Fig. 4 above $15 \mathrm{MeV}$. 


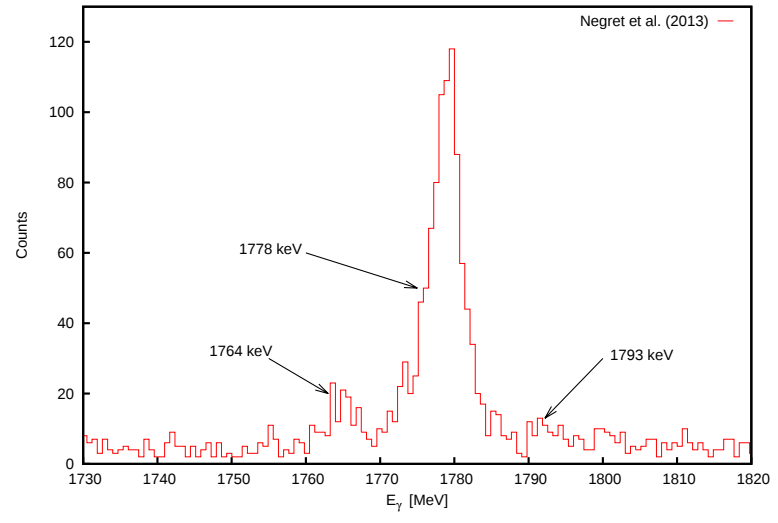

Figure 6. (Colour online) The amplitude spectrum, highlighting the main ${ }^{28} \mathrm{Si}$ transition $(1778.9 \mathrm{keV})$, corresponding to one of the HPGe detectors used in the experiment of Ref. [20]. In the left and the right side of the peak of interest, the neighbouring 1764.4- and 1793.8-keV $\gamma$ peaks are also visible. The spectrum was generated using a $14-18 \mathrm{MeV}$ neutron energy gate.

Further, we constructed similar spectra with the one of Fig. 6, but with incident neutron energies corresponding to $0.5-8$ - and 8-14-MeV ranges, and calculated the ratio given in expression (1). The results yielded a ratio of around $3 \%\left(E_{n}=0.5-8 \mathrm{MeV}\right)$ and 5-7\% $\left(E_{n}=8-14 \mathrm{MeV}\right)$ for the two cases. This fact leads us to conclude that, for the data reported in the present work, the 1764.4- and 1793.8$\mathrm{keV}$ contributions are present in the entire incident energy range but only above $14 \mathrm{MeV}$ they are high enough to generate a noticeable difference between the two data sets displayed in Fig. 4. We also mention that no difference was observed for the secondary ${ }^{28} \mathrm{Si}$ transitions.

With the above explanations, the cross check performed in this section shows a very good agreement between the two experiments. These investigations support the claimed reliability of cross sections measured using the GELINA-GAINS facility.

\section{Conclusions}

Using the GELINA-GAINS setup we measured the neutron inelastic cross section for the $6128.6-\mathrm{keV}$ transition in ${ }^{16} \mathrm{O}$. It is reported with very good neutron energy resolution and very low uncertainty in the entire 6-20 MeV incident energy range. Our results compare very well with other previously reported data. The $\mathrm{SiO}_{2}$ target also allowed to cross check our data by measuring again a few ${ }^{28} \mathrm{Si}$ transitions and by comparing them with a previous experiment performed by our group on the same nucleus. The two data sets mostly agree but there exists a small discrepancy above $15 \mathrm{MeV}$ incident energy which is explained in detail. When taking in consideration this explanation, the overall very good agreement between the two measurements indicates the reliability of the cross sections measured using the GELINA-GAINS facility.

This work was supported by the European Commission within the Seventh Framework Programme through Fission-2013-CHANDA (project no. 605203) and EU-
FRAT (project no. 211499) and by the Ministry of Research and Innovation of Romania, CNCS-UEFISCDI, through the project number PN-III-P4-ID-PCE-2016-0025 within PNCDI III.

\section{References}

[1] https://www.iaea.org/topics/fast reactors (2019)

[2] E. Browne, J. Tuli, Nucl. Data Sheets 127, 191 (2015)

[3] J. Meija, T. Coplen, M. Berglung, W. Brand, P.D. Bievre, M. Groning, N. Holden, J. Irrgeher, R. Loss, T. Walczyk et al., Pure Appl. Chem. 88 (2016)

[4] M. Chadwick, et al., Nuclear Data Sheets 148, 189 (2018)

[5] Nuclear Data High Priority Request List: http://www.oecd-nea.org/dbdata/hprl/, $\quad{ }^{16} \mathrm{O}$ entry. (2014)

[6] W. Mondelaers, P. Schillebeeckx, Notiziario neutroni e luci di sincrotone 11 (2010)

[7] D. Deleanu, C. Borcea, P. Dessagne, M. Kerveno, A. Negret, A. Plompen, J. Thiry, Nucl. Instrum. Methods Phys. Res. A 624 (2010)

[8] A. Olacel, C. Borcea, P. Dessagne, M. Kerveno, A. Negret, A. Plompen, Physical Review C 90 (2014)

[9] A. Olacel, F. Belloni, C. Borcea, M. Boromiza, P. Dessagne, G. Henning, M. Kerveno, A. Negret, M. Nyman, E. Pirovano et al., Physical Review C 96 (2017)

[10] C. Rouki, P. Archier, C. Borcea, C. de Saint Jean, J. Drohe, S. Kopecky, A. Moens, N. Nankov, A. Negret, G. Noguere et al., Nucl. Instrum. Methods Phys. Res. A 672 (2012)

[11] C. Brune, Nucl. Instrum. Meth. A 493 (2002)

[12] L. Mihailescu, PhD thesis, European Commission Joint Research Center Geel, Belgium (2006)

[13] L. Mihailescu, C. Borcea, A. Plompen, Nucl. Instrum. Methods Phys. Res. A 578, 298 (2007)

[14] M. Boromiza, PhD thesis, University of Bucharest, Romania (2018)

[15] D.R.Tilley, H.R.Weller, C.M.Cheves, Nucl. Phys. A564 1 (1993)

[16] R. Nelson, M. Chadwick, A. Michaudon, P. Young, Nuclear Science and Engineering 138, 105-144 (2001)

[17] J. Dickens, F. Perey, Nuclear Science and Engineering 40, 283 (1970)

[18] V. Orphan, C. Hoot, J. John, Nuclear Science and Engineering 42, 352 (1970)

[19] V. Besotosnyj, V. Gorbachev, L. Suvorov, M. Shvecov, Rept: Yadernye Konstanty 19, 77 (1975)

[20] A. Negret, C. Borcea, D. Deleanu, P. Dessagne, D. Filipescu, D. Ghita, T. Glodariu, M. Kerveno, N. Marginean, R. Marginean et al., Phys. Rev. C 88 (2013) 